\title{
Exploiting the complementarity between X-ray scattering and absorption techniques to investigate breast cancer metastasis
}

\author{
Andre L. C. Conceição ${ }^{1}$, Sylvio Haas ${ }^{1}$, Volkmar Müller ${ }^{5}$, Eike Burandt ${ }^{3}$, Malte Mohme ${ }^{4}$ \\ ${ }^{1}$ DESY, Hamburg, Germany; \\ ${ }^{2}$ Universitätsklinikum Hamburg-Eppendorf, Martinistraße 52, D-20246 Hamburg, Germany; \\ andre.conceicao@desy.de
}

Breast cancer is the leading cause of cancer death in women worldwide. Systemic changes in the elemental composition of the microenvironment between the cancer cells and the host stroma play an important role in supporting the growth and progression of the tumor. Excessive accumulation of the trace elements $\mathrm{Fe}, \mathrm{Zn}$, and $\mathrm{Cu}$ and its relationship with the matrix of the tumor microenvironment remodeling has been reported. Although the knowledge of breast carcinogenesis is being progressively elucidated with 2D cell-culture experiments, they are not able to reproduce the real physiological pattern of the tumor microenvironment where the surroundings cells are equally as important as the tumor cell itself. X-ray fluorescence (XRF) has been successfully exploited to detected trace elements in breast tissues, nevertheless, this technique is not sensitive to light elements such as carbon and oxygen, the major constituents of the breast tissue matrix. This information can be complemented by using the Rayleigh-to-Compton ratio technique $(\mathrm{R} / \mathrm{C})$. Likewise, the microenvironment remodeling comprises collagen fibrils rearrangements which can be investigated by Small-angle X-ray scattering (SAXS). Therefore, it will be shown the results of a pilot experiment exploiting the complementarity of the X-ray scattering and spectroscopy signals tomographically acquired, to map three-dimensionally the changes due to cancer progression.

Keywords: Breast cancer, SAXS, XRF, Compton 